WellBeing International

WBI Studies Repository

4-2010

\title{
A Modular One-Generation Reproduction Study as a Flexible Testing System for Regulatory Safety Assessment
}

\author{
Richard Vogel \\ Troy Seidle \\ Humane Society International \\ Horst Spielmann \\ Freie Universitat Berlin
}

Follow this and additional works at: https://www.wellbeingintlstudiesrepository.org/acwp_arte

Part of the Animal Experimentation and Research Commons, Animal Studies Commons, and the Other Medical Sciences Commons

\section{Recommended Citation}

Vogel, R., Seidle, T., \& Spielmann, H. (2010). A modular one-generation reproduction study as a flexible testing system for regulatory safety assessment. Reproductive Toxicology, 29(2), 242-245.

This material is brought to you for free and open access by WellBeing International. It has been accepted for inclusion by an authorized administrator of the WBI Studies Repository. For more information, please contact wbisr-info@wellbeingintl.org.

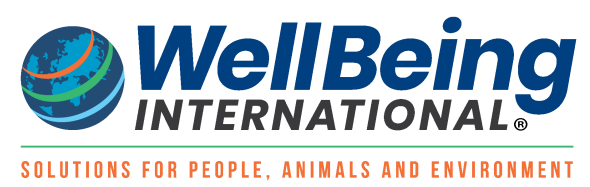




\title{
A modular one-generation reproduction study as a flexible testing system for regulatory safety assessment
}

\author{
Richard Vogel ${ }^{a}$, Troy Seidle ${ }^{b}$, Horst Spielmann $^{c}$ \\ aLeubnitzer Weg 3c, 13593 Berlin, Germany \\ ${ }^{\mathrm{b}}$ Humane Society International/Humane Society Legislative Fund/Humane Society of the United States, \\ Toronto, Canada \\ ${ }^{\mathrm{c}}$ Freie Universität Berlin, Faculty of Biology, Chemistry, Pharmacy, Berlin, Germany
}

\section{CITATION}

Vogel, R., Seidle, T., \& Spielmann, H. (2010). A modular one-generation reproduction study as a flexible testing system for regulatory safety assessment. Reproductive Toxicology, 29(2), 242-245.

\section{KEYWORDS}

reproduction toxicity, developmental toxicity, neurotoxicity, immunotoxicity, generation study, testing strategy, OECD

\section{ABSTRACT}

The European Union's Registration, Evaluation and Authorisation of Chemicals (REACH) legislation mandates testing and evaluation of approximately 30,000 existing substances within a short period of time, beginning with the most widely used "high production volume" (HPV) chemicals. REACH testing requirements for the roughly $3000 \mathrm{HPV}$ chemicals specify three separate tests for reproductive toxicity: two developmental toxicity studies on different animal species (OECD Test Guideline 414) and a twogeneration reproduction toxicity study (OECD TG 416). These studies are highly costly in both economic and animal welfare terms. OECD TG 416 is a fertility study intended to evaluate reproductive performance of animals in the $\mathrm{P}$ and F1-generations following repeated exposure to a test substance. It can also be used to detect adverse effects on structural and functional development. Thus, it has conventionally been preferred to the one-generation study (OECD TG 415). Recently, the Agricultural Chemical Safety Assessment (ACSA) Technical Committee of the ILSI Health and Environmental Sciences Institute (HESI) proposed that routine two-generation studies could in most cases be replaced with an "enhanced" one-generation study (Reuter et al. [1]). The flexible design proposed by HESI-ACSA allows for the addition of one or more specialised modules, if triggered (e.g. production of a second generation or the investigation of classical developmental toxicity or developmental neuro- or immunotoxicity). Significantly, however, the HESI-ACSA proposal was designed for use in the safety assessment of pesticidal, as opposed to industrial, chemicals. Thus for the purposes of REACH, a streamlined one-generation study that also examines structural development would be the most efficient means of addressing current information requirements for HPV chemicals. This study represents a flexible testing system that can be modified to meet regulatory needs in a variety of sectors.

\section{Introduction}

Impairment of fertility and toxicity to the developing embryo are the principal endpoints to be determined in regulatory testing for reproductive toxicity. Several internationally accepted Test Guidelines (TG) of the Organisation for Economic Co-operation and Development (OECD) are available for this purpose: TG 414 to detect developmental toxicity, one- and two-generation reproduction studies (TGs 415 and 416 ) to 
detect fertility impairment, and combined reproductive/developmental toxicity screening studies (TGs 421 and 422). While the latter offer certain advantages in terms of reduced costs and animal use, their value for hazard assessment is limited by a number of recognized shortcomings [1]. Thus, the revision of TG 415 for use under the European Union (EU) regulation concerning the Registration, Evaluation and Authorisation of Chemicals (REACH) should be seen as an urgent priority [2]. Our new approach for a revised TG 415 is based on the recommendations of the Agricultural Chemical Safety Assessment (ACSA) Technical Committee of the ILSI Health and Environmental Sciences Institute (HESI) for a new extended one-generation study to be used for pesticide testing [3] (Fig. 1) and on an initiative by the German Federal Institute for Risk Assessment (BfR) for an update of the existing TG 415 for chemicals testing under the new EU chemicals legislation REACH. Although originally submitted to the OECD as separate projects, these initiatives have since been merged, and a single draft TG for an extended onegeneration study is currently under discussion by OECD expert groups. The following presentation is intended to contribute to this international initiative, and in particular, to support the establishment of an OECD TG based on a new streamlined one-generation study that will yield reliable results for classification and labelling of chemicals, but which may also be extended to include one or more modules as a means of gathering needed data as efficiently and economically as possible.

\begin{tabular}{|c|c|c|c|c|c|}
\hline \multirow[t]{2}{*}{ M Pre-X 4w } & & \multicolumn{2}{|c|}{ Post-X: up to 6 weeks } & \multicolumn{2}{|l|}{ M necropsy } \\
\hline & $X: 2 w$ & & & \multirow[b]{2}{*}{ F necropsy (plus non-selected surplus F1 pups) } & \multirow[b]{3}{*}{$\begin{array}{l}\text { Set } 1 \text { : Clinical } \\
\text { pathology and dev. } \\
\text { neurotox }\end{array}$} \\
\hline \multirow[t]{4}{*}{ F Pre-X 2w } & & Gestation & Lactation & & \\
\hline & & & & F1: Post-weaning: up to PND 70 & \\
\hline & & & & F1: Post-weaning: up to PND 70 & \multirow{2}{*}{$\begin{array}{l}\text { Set } 2: \text { Immunotox } \\
\text { Set } 3 \text { :Estrous cycles } \\
\text { and triggered reprotox } \\
\text { or reversibility }\end{array}$} \\
\hline & & & & $\begin{array}{l}\text { F1: Post-weaning: up to PND } 70 \\
\text { (with triggered extension) }\end{array}$ & \\
\hline \multicolumn{4}{|c|}{ Parental animals treatment } & F1 animals treatment & \\
\hline
\end{tabular}

Fig. 1. The HESI-ACSA approach for an extended one-generation study [1].

\section{Added value of a second generation}

A number of retrospective analyses of multigenerational studies have cast doubt upon the longstanding assumption that the production of at least two generations of offspring is necessary for the accurate identification of reproductive toxicants and dose response characterization. For instance:

- A review of approximately 300 pesticide multigenerational studies in the US "SABRE" database revealed that for only two substances would adverse effects not have been detected based on F1 results or other available toxicology data [1]. A detailed publication of these findings is anticipated in the near future.

- The Dutch National Health Authority (RIVM) conducted a retrospective study on 176 multigenerational studies that had been submitted to EU and US authorities [4]. In the twogeneration studies, the effects reported in $\mathrm{F} 2$ animals did not affect the overall NOAEL. It was therefore concluded that the two-generation study impacted neither risk assessment nor classification and labelling. 
- A review of 22 two-generation studies identified three studies in which effects were confined exclusively to the second generation. However, applying the triggers proposed by HESI-ACSA revealed that all three studies would have been extended to the second generation $[3,5]$.

The above examples illustrate that, almost without exception, F2 data do not contribute essential information for risk assessment and regulation beyond what can be gleaned from F1 results, and that in the handful of instances where this is not the case, F1 results would reliably trigger an extension to a second generation. It is essential, that the possibility of omitting a second generation will not impair the safety of consumers and workers. Therefore, all available data from two-generation studies will be analysed once again retrospectively and assessed by using standardized criteria. As a result, it is expected that a routine requirement to conduct two-generation studies should be discontinued in favour of a flexible testing strategy based on a one-generation study.

\section{Proposed new test design}

\subsection{Standard test}

Given the range of potential regulatory applications of an enhanced one-generation study, we recommend that the core design be limited to a one-generation study according to the OECD TG415. This study should be updated in thewaythe two-generation study (OECD TG 416) was updated several years ago. Additional modules may be included to this updated one-generation study depending on triggers and/or sector-specific requirements (Fig. 2). Based on intensive discussions concerning triggering and waiving criteria at an April 2008 expert workshop co-organised by the European Centre for Ecotoxicology and Toxicology of Chemicals (ECETOC) and the European Centre for the Validation of Alternative Methods (ECVAM) [6], at which participants could not reach agreement on reliable criteria for waiving a module, we do not believe that an all-encompassing, waiver-based approach is feasible in current regulatory practice. Therefore, we provide guidance to trigger modules which could be added to the core study.

\subsection{Triggered second mating or second generation module}

If unequivocal positive or negative findings on fertility parameters were obtained from the standard test, no further testing would be necessary. Equivocal results, however, could lead to a more detailed evaluation of a second litter or to a second generation (Fig. 2). This type of flexible approach is already standard practice [7]. To produce a second generation, F1 pups could be selected and processed as described in TG 416.

\subsection{Triggered developmental toxicity module (OECD TG 414 rat)}

We propose that clear evidence of adverse effects upon structural development in the enhanced onegeneration study should permit classification and labelling without further testing. Likewise, if data are clearly negative, further testing would be unlikely to influence a classification or risk assessment decision, since it is improbable that a signal that an agent is a developmental toxicant would be completely overlooked when the entire F1-generation is analysed. However, equivocal results could trigger a prenatal developmental toxicity module or a stand-alone TG 414 study (Fig. 2). 


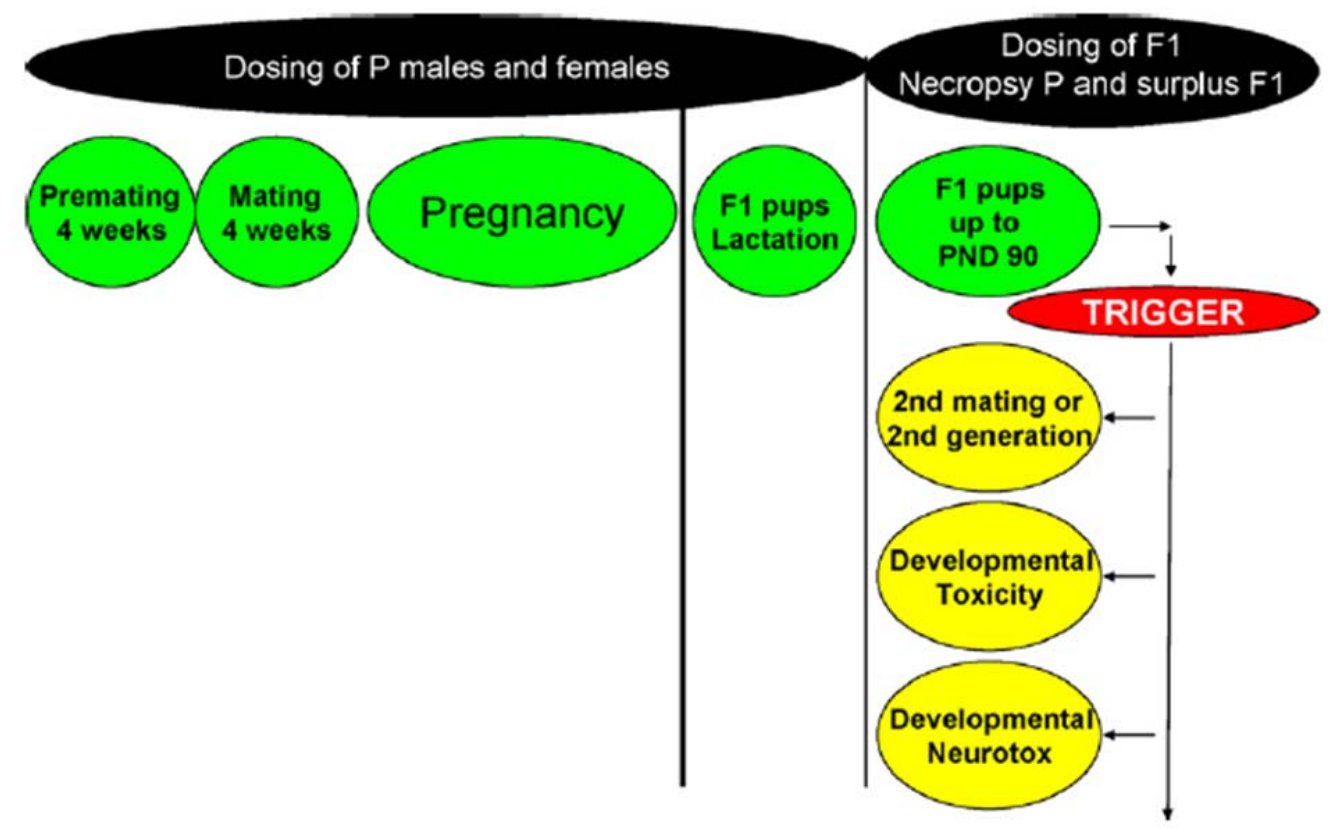

Fig. 2. Our proposal for a flexible, modular one-generation reproduction toxicity study.

\subsection{Triggered developmental neurotoxicity module (OECD TG 426)}

Testing for developmental neurotoxicity (DNT) is not a legal requirement in Europe either for pesticides or other chemical substances; in fact, a category for the classification and labelling of neurotoxicants does not currently exist in the EU. On the other hand, developmental neurotoxic effects could possibly be integrated into a common category "reproductive toxicant". In the US, DNT testing has recently been codified as a conditional data requirement for the registration of conventional pesticides, although published evidence suggests that DNT data seldom afford additional or increased human health protection beyond what is achieved by assessing risks from more conventional toxicity studies. This was evident from a US EPA retrospective review of 12 organophosphate pesticides [8], as well as a more recent HESI survey of 113 reproduction studies of pharmaceutical compounds that included behavioural assessment of F1 animals, which revealed that in only $2.6 \%$ of cases did DNT data alone define the noeffect level [9]. These are of course preliminary findings, which must be confirmed by a broader database. Nevertheless, an even lower percentage could be expected for industrial chemicals or other nonbiologically active substances.

Evidence to date does not support the inclusion of DNT as a routine information requirement or as a default module in an enhanced one-generation study. However, in cases where a study according to TG 426 would otherwise be carried out as a stand-alone according to a regulatory requirement and/or external trigger [10], it would be preferable for such an evaluation to occur as a module to a onegeneration study (Fig. 2). Nevertheless it is still open to discussion in which way the results of such studies can be used for classification and labelling under REACH and the GHS.

\subsection{Triggered developmental immunotoxicity (OECD TG not existing)}

Cooper et al. [3] also proposed the inclusion of amodule to evaluate developmental immunotoxicity (DIT) (Fig. 1). However, we believe it is premature to include a DIT module in the enhanced one-generation TG for the following reasons 
- No regulatory data requirement for DIT exists anywhere in the world, and only a handful of ad hoc "special studies" have been requested to date.

- Only recently has one pesticide regulator codified a requirement for an adult immunotoxicity study, so no contribution can be claimed to further international harmonization of hazard and risk assessment approaches.

- No standardized or validated methodology or OECD TG currently exists for the evaluation of immunotoxicity in either adult or developing animals, although discussions among experts have taken place [11].

- Although an enhanced one-generation study would be suited for general use, DIT data are of dubious relevance outside the pesticides sector.

This perspective was shared by a majority of participants at the April 2008 ECETOC-ECVAM expert workshop [6].

\section{Validation}

When OECD TG 414, 415 and 416 were introduced in 1982, the tests were not formally validated with respect to their reliability or relevance for the human situation. However, after many years of practical experience, they are generally regarded as valid by regulatory authorities. The proposed modular onegeneration study is based on aspects of these TGs and does not involve new endpoints. Therefore, a formal validation should not be required for the core reproduction study, including the extensions to a second litter/generation, as well as for the prenatal developmental and DNT modules.

The practicability of the new approach is currently under investigation in a joint feasibility project between the European Partnership on Alternative Approaches to Animal Testing (EPAA) and the US EPA.

\section{Implementation and triggers}

A merged draft for an extended one-generation reproductive toxicity study was recently placed on the OECD website for discussion. The draft does not clearly differentiate between a core study design and extensions, since it is merely based on the US protocol to meet the testing requirements for pesticides in the US. Therefore, we would like to separate the components of the study as it was initially proposed in the OECD Standard Project Submission Form (SPSF, not publicly available), to create a flexible modular design to be used under REACH: the "core module" would be a standard one-generation study as an update of TG 415. Equivocal results of this part of the test should trigger a second mating or a second generation. If triggered and/or required by regulatory authorities, one or more additional modules could be included to evaluate developmental toxicity and/or DNT. Triggers for these modules have been proposed $[6,7]$, but have yet to achieve international agreement.

A modular one-generation reproductive toxicity study could, in most cases, replace not only the twogeneration study (TG 416), but also the definitive developmental toxicity study (TG 414) and combined reproductive/developmental toxicity screening studies (TGs 421 and 422) prescribed under REACH, and the DNT study (TG 426), which is now being conditionally required by pesticide regulators in the US. Depending upon the regulatory requirements, the approach we have proposed could provide a simple one-generation test or a comprehensive testing system including all major endpoints in reproductive toxicology. A flexible strategy based on a modular testing system will significantly reduce both economic and animal welfare costs under $\mathrm{REACH}$ and in other sectors, while at the same time ensuring that 
commercial products are safe for human use. Therefore, we strongly support the adoption of a flexible, modular one-generation reproduction toxicity test as an OECD TG as swiftly as possible.

\section{Conflict of interest}

The authors declare that there are no conflicts of interests. Study sponsors had no involvement in the preparation or review of this manuscript.

\section{Acknowledgements}

This research was supported by the German Federal Institute for Risk Assessment (BfR), by Humane Society International and its US affiliates, and sponsored through a grant from the Doerenkamp-Zbinden Foundation.

\section{References}

1. Reuter U, Heinrich-Hirsch B, Hellwig J, Holzum B, Welsch F. Evaluation of OECD screening tests 421 (reproduction/developmental toxicity screening test) and 422 (combined repeated dose toxicity study with the reproduction/developmental toxicity screening test). Regulat Toxicol Pharmacol 2003;38:1726.

2. Spielmann H, Vogel R. The extended 1-generation study (OECD 415) as a replacement of the mammalian 2-generation study (OECD 416). AATEX 2008;14:795-8.

3. Cooper RL, Lamb-IV JC, Barlow SM, Bentley K, Brady AM, Doerrer NG, et al. A tiered approach to life stages testing for agricultural chemical safety assessment. Crit Rev Toxicol 2006;36:69-98.

4. Janer G, Hakkert BC, Slob W, Vermeire T, Piersma AH. A retrospective analysis of the twogeneration study: what is the added value of the second generation? Reprod Toxicol 2007;24:97102.

5. Myers DP, Willoughby CR, Bottomley AM, Buschmann J. An analysis of the results from twogeneration reproduction toxicity studies to assess the value of the second (F1) generation for the detection of adverse treatment-related effects on reproductive performance. Reprod Toxicol 2008, doi:10.1016/j.reprotox.2008.06.004.

6. ECETOC-ECVAM [European Centre for Ecotoxicology and Toxicology of Chemicals and European Centre for the Validation of Alternative Methods]. Report of the Workshop on Triggering and Waiving Criteria for the Extended One-Generation Reproduction Toxicity Study, 14-15 April 2008, Barza d'Ispra, Workshop Report No. 12, 2008.

7. Kayser D, Roll R. Strategy of toxicological testing to assess chemicals within the frame of the chemicals act. BgVV-Schriften 1995;1.

8. Makris S, Raffaele K, Sette W, Seed J. A Retrospective Analysis of Twelve Developmental Neurotoxicity Studies Submitted to the USEPA Office of Prevention, Pesticides and Toxic Substances (OPPTS). Washington, DC: US EPA; 1998.

9. Middaugh LD, Dow-Edwards D, Li AA, Sandler JD, Seed J, Sheets LP, et al. Neurobehavioral assessment: a survey of use and value in safety assessment studies. Toxicol Sci 2003;76:250-61.

10. ECETOC [European Centre for Ecotoxicology and Toxicology of Chemicals]. Document No. 45: Triggering and Waiving Criteria for the Extended One-Generation Reproduction Toxicity Study. Brussels: ECETOC; 2008.

11. Holsapple MP, Burns-Naas LA, Hastings KL, Ladics GS, Lavin AL, Makris SL, et al. Proposed testing framework for developmental immunotoxicology (DIT). Toxicol Sci 2005;83:18-24. 